\title{
A Rasch scaling validation of a 'core' near-death experience
}

\author{
Rense Lange ${ }^{1,3}$, Bruce Greyson ${ }^{2}$ and James Houran ${ }^{3,4} *$ \\ 'Southern Illinois University School of Medicine, and Integrated Knowledge \\ Systems, USA \\ ${ }^{2}$ University of Virginia Health System, USA \\ ${ }^{3}$ University of Adelaide, Australia and Integrated Knowledge Systems, USA
}

\begin{abstract}
For those with true near-death experiences (NDEs), Greyson's (1983, 1990) NDE Scale satisfactorily fits the Rasch rating scale model, thus yielding a unidimensional measure with interval-level scaling properties. With increasing intensity, NDEs reflect peace, joy and harmony, followed by insight and mystical or religious experiences, while the most intense NDEs involve an awareness of things occurring in a different place or time. The semantics of this variable are invariant across True-NDErs' gender, current age, age at time of NDE, and latency and intensity of the NDE, thus identifying NDEs as 'core' experiences whose meaning is unaffected by external variables, regardless of variations in NDEs' intensity. Significant qualitative and quantitative differences were observed between True-NDErs and other respondent groups, mostly revolving around the differential emphasis on paranormal/mystical/religious experiences vs. standard reactions to threat. The findings further suggest that False-Positive respondents reinterpret other profound psychological states as NDEs. Accordingly, the Rasch validation of the typology proposed by Greyson (1983) also provides new insights into previous research, including the possibility of embellishment over time (as indicated by the finding of positive, as well as negative, latency effects) and the potential roles of religious affiliation and religiosity (as indicated by the qualitative differences surrounding paranormal/mystical/religious issues).
\end{abstract}

Many adults and children suddenly faced with death report experiencing a distinctive state of consciousness in which their existence seems to be bound neither to a physical body nor to earthly environs (for a review see: Greyson, 2001). These are termed neardeath experiences (NDEs) and may be defined simply as transcendental experiences precipitated by a confrontation with death and which do not seem to be accounted for by our current medical understanding of the dying process (Greyson, 2001; Irwin, 1999). An important aspect of NDEs from a clinical or transpersonal perspective

*Correspondence should be addressed to James Houran, Integrated Knowledge Systems, 60I Park Blvd, \#902 Grapevine, TX 7605I, USA (e-mail: info@iknowsys.org). 
is the enduring effect they often have upon experiencers' subsequent attitudes and values, particularly if death was thought to be imminent and if the NDE included a panoramic life review (Greyson \& Stevenson, 1980). Berman (1974) suggested that the content of the NDE itself motivates the psychological aftereffects, while other research (Pope, 1991, 1994) implies that the impact of NDEs is due fundamentally to their occurrence in a life-threatening context rather than to the content of the experience.

Popular interest in NDEs arguably was prompted by the publication of Moody's (1975) seminal work, Life After Life, which chronicled more than a decade of investigation into approximately 150 case reports. Based on this case collection, Moody (1975) identified a common set of elements and proposed that these constituted a universal or 'core' experience across people of different ages, genders, and cultures. This core experience is defined by the following phenomenology:

- an overwhelming feeling of peace and well-being, including freedom from pain;

- the impression of being located outside one's physical body;

- floating or drifting through darkness, sometimes described as a tunnel;

- awareness of a golden light;

- encountering and perhaps communicating with a 'presence' described by Moody

as a 'being of light';

- a rapid succession of visual images of one's past;

- experiencing another world of much beauty, perhaps meeting there spirits of

deceased relatives and acquaintances with whom one also might communicate.

More rigorous studies (Grey, 1985; Ring, 1980; Sabom, 1982) have generally confirmed these elements, but several authors (Greyson, 2001; Irwin, 1999; Knoblauch, Schmied, \& Schnettler, 2001; Parker, 2001) also note that major questions can be raised concerning the nature and universality of such a 'core' near-death experience.

In particular, the elements identified by Moody (1975) have been described as 'stages' (Ring, 1980) or a 'sequence' of events (Lundahl, 1993). Yet, there is evidence that the elements do not form a strict (i.e. deterministic) hierarchy, but rather a hierarchy of experiences whose occurrence varies probabilistically. For instance, Greyson's (1983) research using the Near-Death Experience scale (NDE scale) found that about $60 \%$ of those reporting NDEs describe reaching the first stage described by Ring (1980), but only $10 \%$ report making it to the last stage. As a result, a given NDE might not feature all elements and such elements need not occur in a fixed order. Furthermore, the specific content of NDEs seems partly to reflect experiencers' expectations and cultural backgrounds. Irwin (1999) and Greyson (2001) thoroughly discuss this finding, as well as the rare reports of frightening or distressing NDEs in which some of the content contrasts dramatically with more traditional reports. Nevertheless, the core elements are still arguably present even in such negatively toned reports (Irwin, 1999). In addition, the occurrence of NDEs seems to depend, not so much on the fact that the experiencers were dying, but rather that they perceived their life to be under serious threat (Gabbard \& Twemlow, 1991; Kellehear, 1996; Stevenson, Cook, \& McClean-Rice, 1989).

The findings presented above indicate that we do not know the degree to which response biases on the part of experiencers have distorted previous findings on NDEs or whether NDEs comprise qualitatively difference experiences across individuals. To complicate matters, previous research suffers from methodological and conceptual pitfalls whose perpetuation undermines the notion of the NDE as a core experience (Greyson, 1999). Most of these pitfalls have their origins in the use of inappropriate methods to establish the hierarchical nature of NDEs. In fact, the very notion that NDEs 
form a hierarchy has not been validated by appropriate means. For this reason the following section describes Rasch (1960/1980) scaling, as this technique provides an integrated approach to address the issues raised above. We refer interested readers to Bond and Fox (2001) for a more extensive introduction to Rasch scaling. For applications of this approach in areas related to the present study, see the work of Lange and colleagues (Houran \& Lange, 2001; Houran, Wiseman, \& Thalbourne, 2002; Lange, Irwin, \& Houran, 2000; Lange \& Thalbourne, 2002; Lange, Thalbourne, Houran, \& Storm, 2000).

\section{The present research}

As we explain in more detail in the Methods section, this study is based on data obtained via Greyson's (1983) Near-Death Experience scale that asks respondents to rate experiential elements of their NDEs on a series of three-point rating scales. In this context, the notion that the NDE scale should define a probabilistic response hierarchy does not simply mean that its items differ with respect to their endorsement rates. Rather, the Rasch scaling requires that the NDEs' intensities form a (latent) quantitative dimension on which each respondent and each item of the NDE scale assume a position (e.g. Bond \& Fox, 2001). These positions reflect respondents' trait levels and the trait level implied by the item, respectively, and together they determine the likelihood of a given response. These item and person locations (also called, item and person measures) are expressed in a common Logit metric (Wright \& Masters, 1982).

Fit of the Rasch model entails that items form a hierarchy that reflects the structure of the variable. To the extent that this structure conforms to expectations, model fit contributes to construct validity by defining a variable's semantics (for a recent discussion, see Lange, Irwin, \& Houran, 2001). Deviations from the Rasch model also inform theory, as this initiates a dialectic process in which researchers are forced to reconsider their expectations as well as their data collection methods (Bond \& Fox, 2001).

We hypothesize that the structure of the NDE Scale agrees with the findings of Moody (1975) summarized above. Further, to establish that NDEs indeed form a meaningful hierarchy, it is hypothesized that the items of the NDE Scale function in accordance with Andrich's (1978) rating scale model or Masters's (1982) partial credit model. The former assumes that all items share the same category structure, whereas the latter allows the category structure to vary across items. However, both formulations imply that higher response categories should have a greater probability of being selected for items with lower locations than for items with higher locations. (Note: It is assumed that higher response categories indicate higher trait levels.) Also, respondents with higher trait levels should be more likely to give higher ratings than are respondents with lower trait levels. Finally, both properties should hold across all respondents, items and response categories. It can be shown (see e.g. Wright \& Masters, 1982) that when these requirements are fulfilled, the resulting variable has the property that the responses of those with lower trait levels are probabilistic subsets of those with higher trait levels. Also, when these properties hold it follows that simple sum-scores can be transformed to yield interval level measures of respondents' trait levels (Wright \& Masters, 1982).

\section{True-NDErs and core experiences}

We noted earlier that Moody (1975) defined NDEs as 'core' experiences whose nature is little affected by external circumstances, at least for those with true NDEs (True-NDErs). Greyson $(1983,1990)$ introduced the notion of 'True-NDErs' and its definition is 
repeated below. Because Rasch scaling distinguishes between a variable's structure and the values it may assume, it provides a means to test empirically the notion of a 'core' NDE. For instance, the item hierarchy defined by the NDE scale should not vary with True-NDErs' ages or gender, even though the intensity of men's and women's and younger and older people's experience may well differ. Further, although True-NDErs clearly differ with respect to the intensity of their near-death experiences, the NDE scale's item hierarchy should be preserved regardless of the intensity of the NDE. To test these hypotheses, variables other than age and gender will also be considered, including: age at time of NDE, age at responding to NDE scale and latency. The notion of NDEs as 'core' experiences further requires that the hierarchy of NDE scale items differs little or not at all for those with weaker vs. stronger NDEs (for an example of this approach in a different context, see McCutcheon, Lange, \& Houran, 2002).

The hierarchy invariances discussed above might not hold for those who did not experience true NDEs. Also, as we noted earlier, it is often assumed that NDEs have profound effects on experiencers' subsequent views of themselves and their world (Greyson \& Stevenson, 1980). Accordingly, we predict that qualitative differences should exist among the item hierarchies obtained for True-NDErs and those obtained for others. The three comparison groups used in this study are described below.

\section{Method}

\section{The Near-Death Experience scale}

The NDE scale was developed through an iterative process from an initial list of 80 phenomenological features reported to be characteristic of NDEs. Analysis of item-total score correlations yielded a 16-item scale, which had face validity and which was highly correlated with other measures of NDEs. Moreover, this scale differentiated unequivocal NDEs from ambiguous or questionable experiences. Each of the 16 experiences is rated in terms of three ordered categories which generically represent 'not present,' 'mildly or ambiguously present' or 'definitively present', but whose exact wording varies depending on the nature of questions (see Appendix). For instance, the response options for item 3 ('Did scenes from your past come back to you?') are 'No', 'I remembered many past events' and 'My past flashed before me, out of my control'. By contrast, the options for item 10 ('Did you seem to be aware of things going on elsewhere, as of by ESP?') are 'No', 'Yes, but the facts have not been checked out ' and 'Yes, and the facts have been checked out'. When such categories are scored 0,1 and 2 a sum-score of 32 can be achieved. In earlier research (Greyson, 1983) a sum of 7 was chosen as the criterion for identifying someone with NDE because that value was one standard deviation below the mean among a criterion group of NDErs. This approach was later validated in a comparison between NDErs and people who had come close to death without an NDE (Greyson, 1990).

\section{Respondents}

Respondents consisted of a sample of 292 individuals who had contacted the second author in order to share their accounts of their near-death experiences, following media reports of his research. They included 113 men and 179 women, with a mean age of 50.8 years $(M d n=50.0, S D=13.6$, range $=22-92$ years $)$. The largest and most important group consists of 203 NDErs, i.e. respondents who claimed to have had an NDE and whose raw scores $(R)$ on the Near-Death Experience scale were at least 7. 
Three additional groups of respondents were distinguished: (1) Non-NDErs $(n=52)$, those who came near death but denied having had an NDE, and $R<7$; (2) 'False-Positives' $(n=19)$, those who claimed to have had an NDE, but $R \geq 7$; and (3) 'False-Negatives' $(n=17)$, those who denied having had an NDE, but $R>7$. The average age at the time of the NDE was 31.6 years $(M d n=30.0, S D=15.3)$ and the average number of years elapsed since the NDE was $19.3(M d n=15.0, S D=15.2)$.

\section{Rasch scaling}

Facets (Linacre, 2001) and Winsteps (Linacre \& Wright, 1998) software were used to estimate the Logit values of the item and person measures from the empirical data, as well as to determine the tenability of the Rasch assumptions and the reliability of the item and person measures. The sole fit statistic reported in the following is items' Outfit (cf. Wright \& Masters, 1982). Items' optimal Outfit value is 1 , but values in the range 0.6-1.4 are generally deemed acceptable (Bond \& Fox, 2001). All tests for item shifts are based on Facets as this software provides omnibus chi-square tests across all items simultaneously, as well as item-specific tests of statistical significance.

\section{Preliminary analyses}

Recall that the 16 items from the NDE scale use different response formats, each providing three ordered choices. Although a partial credit model (Masters, 1982) approach was considered as well, preliminary analyses indicated that respondents' ratings could satisfactorily be treated as if a common three-point scale had been used. Thus, Andrich's (1978) rating scale model was adopted throughout as this simplifies the analyses and the presentation of the results.

\section{Results}

Because the scaling of the NDE scale items is primarily relevant for those with true NDEs, we first analysed the data of those who reported NDEs and who summed ratings are $\geq 7$. Next, the results of this group were compared with those of the remaining respondent groups. Throughout, the analyses emphasized the structure of NDEs, i.e. the focus was on the NDE scale's items and their structure, while group mean differences were of secondary interest.

\section{True-NDErs}

Indicative of unidimensionality and model fit, the data of the True-NDErs fit the Rasch model satisfactorily. The left-handside of Table 1 shows that 3 of the 16 NDE scale items are somewhat noisy (i.e. their Outfit values exceed the criterion of 1.4), but none of the 16 Outfit values significantly exceeds the optimal value of 1 (all $p>.01$ ). Accordingly, for True-NDErs the items indeed define a meaningful hierarchy that satisfies the measurement requirements of the Rasch model sufficiently for our purposes. This hierarchy is non-trivial as items' locations (Column 1) differ significantly $\left(\chi^{2}(15)=906.4, p<.001\right)$.

The item hierarchy is shown along the $y$-axis in Fig. 1, which plots items according to their estimated locations in Logits. Note that the hierarchy validates the 'core' set of elements identified by Moody (1975), and their sequence in the Rasch hierarchy corresponds to the 'stages' of NDEs argued by Ring (1980) and Lundahl (1993). In 







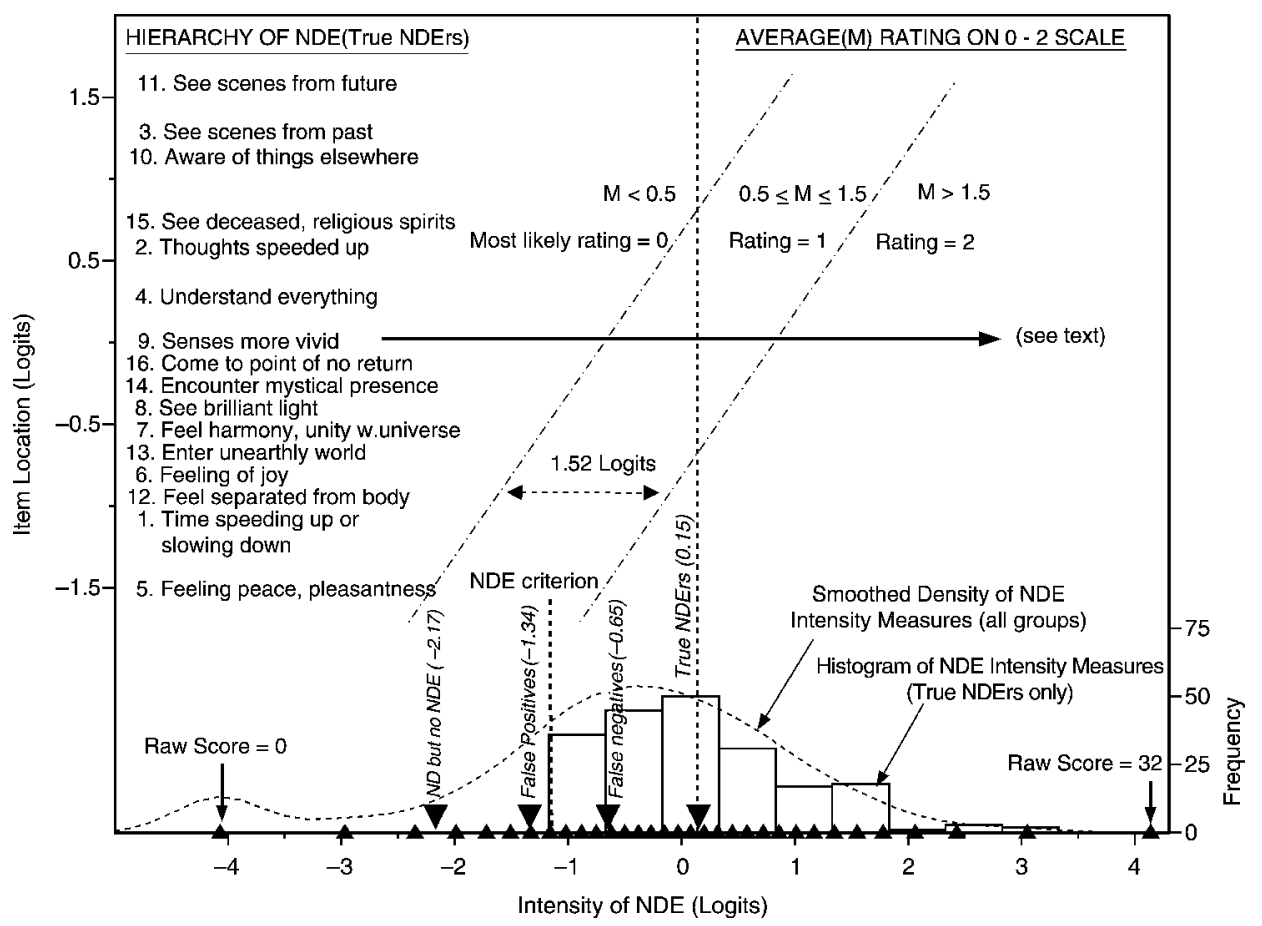

Figure I. Item hierarchy for True-NDErs and intensity distributions.

particular, experiences of peace, joy and harmony occur first (lowest) in the hierarchy, followed by insight and paranormal (i.e. mystical or religious) experiences, and finally an awareness of things occurring in a different place or time. We point out that the most salient features of experiencers' NDEs-extrasensory and supernaturalistic elements (Kelly, Greyson, \& Stevenson, 1999)-occur quite late in the hierarchy. It is stressed that experiences at higher locations do not replace those at lower locations. Rather, the hierarchical structure implied by the Rasch model entails that stronger elements of NDEs (top of hierarchy) are likely reported only in conjunction with reports of more common elements of NDEs (bottom).

The intensity of respondents' NDEs varies along the $x$-axis, and, as indicated by the slanted lines, so do items' average ratings. By following the vertical (dotted) line it can be seen that respondents with experiences of average intensity $(0.15$ Logits $)$ most likely rate experiences reflected in items 5, 1, 12, 6 and 13 as ' 2 ' (definitely present), items 7, $8,14,16,9,4,2$ and 15 as ' 1 ' (mildly or ambiguously present), and items 10, 3 and 11 as ' 0 ' (not present). As illustrated by the horizontal arrow for the case of item 9 ('senses more vivid'), the modelled average measures for these categories increase with the intensity of respondents' NDEs. For example, for item 9 those with NDE intensities up to approximately -0.75 Logits have an average rating below 0.5 on the $0-2$ rating scale, this average ranges from 0.5 to 1.5 for intensities between -0.75 and 0.75 Logits, and it exceeds 1.5 for NDE with an overall intensity $>0.75$.

\section{Logits}

The reader can derive the analogous information for each of the other items by drawing a horizontal line at items' locations ( $y$-axis) and inspecting the $x$-intersections with the 
two slanted lines in the center of the figure. We further note that, depending on the intensity of respondents' NDE, increasing respondents' average category ratings from $<0.5$ (i.e. most likely 0 ) to $>1.5$ (most likely 2 ) would require that their NDE intensity increases by at least 1.52 Logits.

\section{Person measurement}

As was the case for the items, the answer patterns of the respondents fit the Rasch model well. The proportion of response records that deviate from the Rasch model (0.04) does not exceed chance level ( $p<.05$, two-sided) and the rating categories show an acceptable Outfit (range: $0.96-1.24$ ). The person measures span a range of about 8 Logits and the histogram in the bottom of Fig. 1 illustrates that the distribution of these measures is truncated at the lower end (as is required by the definition of True-NDErs, see Method). The triangles along the $x$-axis show the nonlinear (i.e. logistic) correspondence between NDErs raw scores (i.e. the summed rating scales) and the intensity of their NDEs as estimated via maximum likelihood methods. The raw score to Rasch trait level conversion is also listed in Table 2 together with the local (i.e. level specific) standard errors of measurement. As is indicated in Table 2, as well as by the vertical line labelled 'NDE criterion' in Fig. 1, the cut-off for identifying True-NDErs (sum-score $=7$ ) corresponds approximately to a Rasch person measure of -1.25 Logits.

\section{Main effects}

Although subgroups of True-NDErs may differ with respect to the intensity of their experiences, the NDE Scale's item hierarchy (and, hence, the meaning of the items) should be universal. To address this assumption, in addition to gender, four other respondent variables were considered: age at time of NDE, age when responding to the NDE Scale, time between NDE and administration of NDE Scale (latency) and the intensity of NDE. With respect to these last four variables the respondents were divided into two groups based on the median values shown in the first column of Table 3 . Because the five grouping variables discussed above define a total of 64 cells, many cells contained just one case and some were empty. Hence, to maintain acceptable cell sizes each of the five variables was addressed separately using one-way analyses.

We first compared the intensity of the NDE (in Logits) across each respondent variable. Table 3 shows the average group effects in Logits and significance tests as obtained via Facets, as well as the effect size (i.e. in terms of standard deviation of respondents' Rasch measures). It can be seen that the women reported slightly less intense NDEs than did the men, but the effect is not statistically significant and neither is the effect of age at time of NDE. However, age at the time of responding to the NDE scale $(0.32 S D)$, as well as the latency between experiencing and responding $(0.25 S D)$, showed significant effects. Specifically, when reported at a later age (50 years or older) NDE appear more intense then when reported earlier ( 49 or younger), and the intensity of the reported NDE increased with their latency (shorter vs. longer than 15 years).

\section{Hierarchy invariance}

Next, we tested whether NDEs are indeed 'core' experiences whose meaning is invariant across the groups listed in Table 3. Despite the main effects reported above, the rightmost column of this table shows no significant hierarchy differences for any of the five grouping variables. In other words, respondents' gender, their age at the time of NDE or reporting NDE and the latency did not significantly change the item hierarchy as 
Table 2. Translation of raw sums to estimated Rasch person measures based on True-NDErs' data

\begin{tabular}{|c|c|c|c|}
\hline Raw sum & Person measure $(\theta)^{\mathrm{a}, \mathrm{b}}$ & Local $\mathrm{SE}_{\theta}{ }^{\mathrm{a}}$ & \\
\hline 0 & $-4.07^{b}$ & - & Non-NDErs + False-Positives \\
\hline 1 & -2.97 & 0.94 & \\
\hline 2 & -2.35 & 0.67 & \\
\hline 3 & -1.99 & 0.55 & \\
\hline 4 & -1.72 & 0.49 & \\
\hline 5 & $-|.5|$ & 0.44 & \\
\hline 6 & -1.33 & 0.41 & \\
\hline 7 & -1.16 & 0.39 & True-NDErs+False-Negatives \\
\hline 8 & -1.02 & 0.38 & \\
\hline 9 & -0.88 & 0.37 & \\
\hline 10 & -0.75 & 0.36 & \\
\hline II & -0.62 & 0.35 & \\
\hline 12 & -0.50 & 0.35 & \\
\hline 13 & -0.38 & 0.34 & \\
\hline 14 & -0.27 & 0.34 & \\
\hline 15 & -0.15 & 0.34 & \\
\hline 16 & -0.03 & 0.34 & \\
\hline 17 & 0.08 & 0.34 & \\
\hline 18 & 0.20 & 0.35 & \\
\hline 19 & 0.32 & 0.35 & \\
\hline 20 & 0.45 & 0.36 & \\
\hline 21 & 0.58 & 0.36 & \\
\hline 22 & 0.72 & 0.37 & \\
\hline 23 & 0.86 & 0.38 & \\
\hline 24 & 1.01 & 0.40 & \\
\hline 25 & 1.17 & $0.4 I$ & \\
\hline 26 & 1.35 & 0.43 & \\
\hline 27 & 1.55 & 0.46 & \\
\hline 28 & 1.78 & 0.50 & \\
\hline 29 & 2.06 & 0.56 & \\
\hline 30 & 2.43 & 0.67 & \\
\hline 31 & 3.05 & 0.94 & \\
\hline 32 & $4.14^{b}$ & - & \\
\hline
\end{tabular}

${ }^{\mathrm{a}}$ In Logits.

${ }^{\mathrm{b}}$ Projected value.

a whole (all $p$ 's $>.40$ ). Most importantly, the test for hierarchy differences was not significant for low- vs. high-scoring respondents $\left(\chi^{2}(32)=36.7, p>.25\right)$ and the correlation between the 16 items' locations in the low and high groups was extremely high $(r=.95, p<.001)$. Thus, the large difference in the intensity of low vs. high respondents' NDE (1.36 SD) did not systematically alter respondents' interpretation of the NDE Scale items. It follows, therefore, that True-NDErs' responses preserve the probabilistic item hierarchy shown in Fig. 1 regardless of the intensity of their NDE.

The only qualification to be made is that some isolated items differed significantly $(p<.01)$ across the grouping variables discussed above. Notably, those with intense NDEs disproportionately endorsed items 1 ('time speeding up or slowing 
Table 3. Analyses of main effects and item shifts (True-NDErs only)

\begin{tabular}{|c|c|c|c|c|c|c|}
\hline \multirow[b]{3}{*}{$\begin{array}{l}\text { Respondent } \\
\text { characteristic }\end{array}$} & & \multirow{2}{*}{\multicolumn{4}{|c|}{ Intensity differences }} & \multirow{3}{*}{$\begin{array}{c}\text { Hierarchy differences } \\
\text { Overall item shifts } \\
\text { across groups } \\
\chi^{2}(32)\end{array}$} \\
\hline & & & & & & \\
\hline & & Women & Men & $\begin{array}{c}\text { Main effect } \\
\chi^{2}(I)\end{array}$ & $\begin{array}{l}\text { Effect size } \\
\quad \text { SD }\end{array}$ & \\
\hline \multirow[t]{2}{*}{ Gender } & & $-0.04^{\mathrm{a}}$ & 0.04 & 1.5 & $-0.09^{\mathrm{a}}$ & 14.6 \\
\hline & Median & Below & Above & & & \\
\hline $\begin{array}{l}\text { Age at time } \\
\text { of NDE }\end{array}$ & 30 years & -0.02 & 0.02 & 0.9 & -0.05 & II.4 \\
\hline $\begin{array}{l}\text { Age at time of } \\
\text { administration of } \\
\text { NDE scale }\end{array}$ & 50 years & -0.14 & 0.14 & $36.6 *$ & -0.32 & 32.4 \\
\hline Latency & 15 years & -0.11 & 0.11 & $20.4^{*}$ & -0.25 & 10.8 \\
\hline $\begin{array}{l}\text { Intensity } \\
\text { of NDE }\end{array}$ & 0.08 Logits & -0.59 & 0.59 & $585.3^{*}$ & -1.36 & 36.7 \\
\hline
\end{tabular}

${ }^{*} p<.01$.

${ }^{\text {a } A l l ~ m e a n s ~ a n d ~ e f f e c t ~ s i z e s ~ a r e ~ e x p r e s s e d ~ i n ~ L o g i t s . ~}$

down'), 10 ('awareness of things elsewhere'), and 16 ('coming to point of no return') relative to those with less intense NDEs. However, as these effects did not significantly impact the hierarchy as a whole they were not interpreted further.

\section{NDErs vs. other groups}

In a second set of analyses the item hierarchy of the True-NDErs was compared with that obtained for the other three respondent groups, i.e. respondents who did not claim NDE (Non-NDErs), those classified as False-Negatives, or as False-Positives (see Methods). Given the definitions of the four subgroups it is not surprising to find that the mean intensity of their NDE differs significantly $\left(\chi^{2}(3)=743.3, p<.001\right)$, and all pairwise mean differences are statistically significant $(p<.01)$. As indicated by the large (labelled) triangles in Fig. 1, on average, True-NDErs show the most intense experiences $(M=0.15)$, followed by the False-Negative $(M=-0.65)$, False-Positive $(M=-1.34)$ and Non-NDE groups $(M=-2.17)$ (all values are in Logits).

The slight bimodality in the distribution of all respondents (bottom of dotted curve in Fig. 1) is due to the fact that 23 of the 52 Non-NDErs (i.e. 44\%) did not endorse a single item. By contrast, all of the False-Positives (i.e. the only other group that possibly could have a raw score of 0 ) endorsed at least one item (Cramer's $V=0.42, p<.001$ ). Figure 1 further shows that among the most sensationalized features of NDEs, i.e. encountering mystical presences or deceased or religious spirits (items 14 and 15), are reported mostly by True-NDE and False-Negative respondents. Non-NDErs and FalsePositives by contrast most commonly rate these items as 0 .

As we anticipated, the item hierarchy varies significantly across the four subgroups $\left(\chi^{2}(64)=105.4, p<.01\right)$. Figure 2 illustrates that the 16 item locations obtained in the Non-NDE, False-Negative and False-Positive groups respectively correlate just $.64, .73$ and .79 with those obtained for the True-NDErs. Please note that each of these values is considerably lower than the .95 correlation between the items' locations of low vs. high-scoring True-NDErs reported above (all $p<.10$ ). Detailed analyses were performed to study the implications of these finding. The rightmost side of Table 1 


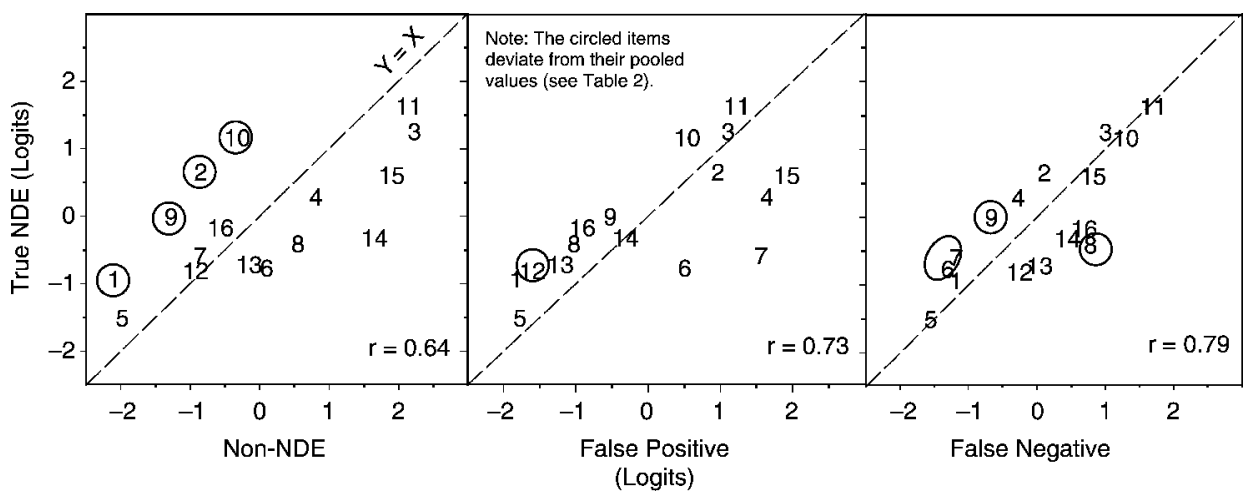

Figure 2. Item locations in the Non-NDE, False-Negative and False-Positive groups vs. those obtained for True-NDErs.

shows the shifts in items' locations in each subgroup relative to the item location obtained by combining all respondents' data. These shifts quantify the difference between items' locations within subgroups and that obtained for all respondents. Negative shifts indicate that items received disproportionately many bigh ratings in a particular group relative to the other groups, and these items' locations are lower than their common values by the amounts shown. Positive shifts reflect that items received disproportionately many low ratings and that these items' locations lie above the common locations.

\section{Non-NDErs}

Those who came near death (but did not report NDEs) over-endorsed items dealing with fairly objective issues at the expense of items related to subjective and mystical experiences. In particular, the negative values in Column 5 of Table 1 indicate that Non-NDErs gave disproportionately bigh ratings to items 1, 2, 9 and 10. This is also reflected in Fig. 2 which shows that these four items lie above the line $Y=X$, i.e. they are more 'easily' endorsed by non-NDErs than by True-NDErs with similarly intense NDE. Note that items 1, 2, 9 and 10 address fairly objective experiences such as time speeding up or slowing down, thoughts speeding up, increased vividness of the senses and being aware of things going on elsewhere, respectively. By contrast, relative to their trait levels, Non-NDErs under-reported 'scenes from the past and the future' (items 3 and 11), 'seeing brilliant lights' (item 8), 'understanding everything' (item 4), 'feeling joy' (item 6) and 'entering another world' (item 13). They also gave disproportionately low ratings to item 14, which mentions 'mystical beings or presences' and item 15, 'seeing religious spirits', Non-NDErs' tendency to under-endorse items 3, 4, 6, 8, 11, 13, 14 and 15 is quite pronounced $(M=0.49)$, resulting in a considerable upward item shift $(t(15)=1.96, p<.10)$ (i.e. these items lie below the line $Y=X)$. These findings imply that Non-NDErs' under-report experiences with religious and mystical elements.

\section{False-Negatives and False-Positives}

The key to the item shifts for respondents who claimed to have had an NDE but scored low (False-Positives) and those who denied having had an NDE but scored high (FalseNegatives) lies in the considerable negative correlation $(r=-.62)$ among the values in 
Columns 6 and 7 of Table 1. Given this pattern of item shifts it is not surprising that the item locations for the False-Positive and False-Negative groups show a very low correlation only $(r=.34)$ (see Fig. 3). In other words, these two groups assign different semantics to the items in the NDE Scale (cf. Lange et al., 2001), thereby suggesting that their responses refer to different experiences.

The two groups' contrasting interpretations involve items 14 and 15 dealing with 'religious spirits' and 'mystical beings', and even more strongly 'feelings of joy' (item 6), 'sense of harmony with universe' (item 7) and 'understanding everything' (item 4). These items lie below the line $Y=X$ in Fig. 3 indicating that False-Positive respondents reject these items disproportionately (positive item shift), while False-Negative respondents disproportionately embrace these items (negative shift). By contrast, items 8 ('seeing brilliant lights'), 10 ('being aware of things elsewhere'), 12 ('feeling separated from the body'), 13 ('enter other, unearthly world') and 16 ('coming to a point of no return') are over-endorsed by the False-Positives (negative shifts) and underendorsed by the False-Negatives (positive shifts). We note that some of these last items partly characterize perceptions related to depersonalization, dissociation, and complex partial epileptic-like symptom clusters. Therefore, the pattern of over-reporting by False-Positive respondents allows for the possibility that these respondents mistook other profound psychological states for NDEs.

\section{Ancillary analyses}

The finding that the reported intensity of True-NDErs' experiences increases with latency contradicts research by Alvarado and Zingrone (1997) who studied respondents similar to our True-NDErs. Lester (2003) similarly reported null effects of latency on depth of NDEs, as measured by Ring's (1980) Weighted Core Experience Index. As these studies suggest that recollections of NDEs are not significantly embellished over time, we compared low- vs. high-latency respondents in the Non-NDE, False-Positive and

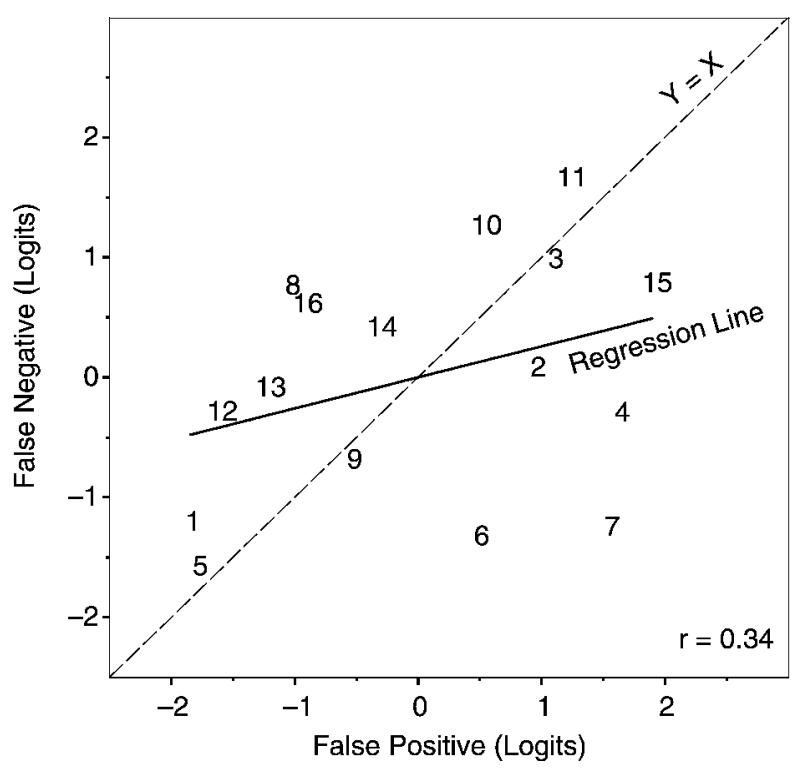

Figure 3. Item locations in the False-Negative group vs. item locations in the False-Positive group. 
False-Negative groups as well. In contrast to the increase shown by True-NDErs, the Non-NDE, False-Positive and False-Negative groups each showed a decrease in NDE intensity with greater latency. The latency effects in these three groups are -0.09 , -0.24 and -0.36 Logits, respectively, and the last decrease (False-Negative group) differs significantly from $0(z=-2.21, p<.05)$. Although our findings do not agree with those of Alvarado and Zingrone (1997) and Lester (2003), we note that all studies thus far are cross-sectional. As such, a definitive resolution of the question of embellishment over time may have to await longitudinal research.

\section{Discussion}

Our results indicate that-at least for those with true NDEs as defined here-Greyson's (1983, 1990) NDE scale satisfactorily fits the Rasch model, thus yielding a scale with interval-level measurement properties. Construct validity is supported by the finding that the item hierarchy agrees with the characterization of NDEs by Ring (1980) and Lundahl (1993). That is, the item hierarchy obtained for True-NDErs shows a probabilistic progression in which experiences of peace, joy and harmony occur most often, followed by insight and mystical or religious experiences, whereas the most intense NDEs reflect an awareness of things occurring in a different place or time. This hierarchy is invariant across True-NDErs' gender, current age, age at time of NDE and latency, even though these last two variables showed considerable main effects on the intensity of the NDE. Accordingly, our results challenge previous work suggesting that true NDEs are multi-dimensional (Lester, 2000; Sabom, 1982) and that personal and circumstance-related variables necessarily influence major elements of the NDE, such as transcendental environment, life review, subjective sense of death and sense of bodily separation (Lester, 2000). As we anticipated, the item hierarchy was highly similar for True-NDErs with weak vs. strong NDE $(r=.95)$, despite the fact that the intensity of their NDEs differed by more than 1.3 SD. Thus, NDEs indeed appear to form a 'core' experience whose basic structure and semantics are preserved regardless of demographic differences and extreme variation in the intensity of NDE.

The validity of the group distinctions proposed by Greyson $(1983,1990)$ received strong support from the finding of hierarchy differences among the True-NDE, FalsePositive, False-Negative and Non-NDE groups. Thus, in addition to quantitative differences, these four groups also exhibit qualitative differences. In particular, the findings suggest that the False-Negative group under-reported paranormal/mystical/ religious experiences, whereas the False-Positive group over-reported such experiences. The hierarchy shifts further support the hypothesis that False-Positives erroneously reinterpreted other intense psychological states as NDEs. This interpretation is consistent with Houran and Lange's (1997) conclusion that the ambiguous experiences that may accompany the dying process are reinterpreted according to the context in which they occur, as well as the finding that beliefs form a threshold for such experiences (Lange \& Houran, 1997). If so, the False-Positive group's disproportionate endorsement of paranormal topics agrees with the attribution model of paranormal belief and experience proposed by Lange and Houran (1998, 1999). This may help explain why specific contents of NDEs seem to correspond to the prevailing cultural and situational context of experients (Greyson, 2001; Irwin, 1999).

Together with the contradictory findings by Alvarado and Zingrone (1997) and Lester (2003), the above implies that different results may be obtained depending on the respondent group being studied (cf. Greyson, 1999). We are particularly intrigued by 
the fact that over $40 \%$ of the Non-NDErs did not endorse a single item of the NDE Scale, i.e. some who came near death could not express their experiences on this scale. The question thus arises how such individuals' experiences should be characterized and studied. Already, the present findings indicate that Non-NDErs over-report experiences that resemble standard reactions to perceived threat. Hence, the inclusion of contextualand personality-related variables (for a review see: Irwin, 1999; 2000) in future studies with our new Rasch scoring scheme for Greyson's (1983, 1990) NDE Scale (cf. Table 2) may help resolve outstanding issues concerning true NDEs that were not the focus of the present research. In particular, is it the content of NDEs or the context of these experiences that motivates psychological after-effects in experiencers? And, are NDEs inherent only to the dying process or do they manifest in tandem with situations that are merely perceived as life-threatening? We believe our findings clearly demonstrate that Rasch scaling provides a powerful aid in the pursuit of these and other pertinent questions. In this way, we can broaden our understanding of all experiences that are associated with coming near death.

\section{Acknowledgments}

We thank the reviewers for their helpful feedback. An earlier version of this paper was presented at the 21st Annual Meeting of the Society for Scientific Exploration, 28 May-1 June 2002, University of Virginia, Charlottesville, VA, USA.

\section{References}

Alvarado, C., \& Zingrone, N. L. (1997). Factors related to the depth of near-death experiences: Testing the 'embellishment over time' hypothesis. Imagination, Cognition \& Personality, 17 , $339-344$.

Andrich, D. (1978). Scaling attitude items constructed and scored in the Likert tradition. Educational and Psychological Measurement, 38, 665-680.

Berman, A. L. (1974). Belief in afterlife, religion, religiosity and life-threatening experiences. Omega, 5, $127-135$.

Bond, T. G., \& Fox, C. M. (2001). Applying the Rasch model: Fundamental measurement in the buman sciences. Mahwah, NJ: Erlbaum.

Gabbard, G. O., \& Twemlow, S. W. (1991). Do 'near-death experiences' occur only near death? Revisited. Journal of Near-Death Studies, 10, $41-47$.

Grey, M. (1985). Return from death: An exploration of the near-death experience. London: Arkana.

Greyson, B. (1983). The Near-Death Experience Scale: Construction, reliability, and validity. Journal of Nervous and Mental Disease, 171, 369-375.

Greyson, B. (1990). Near-death encounters with and without near-death experiences: Comparative NDE Scale profiles. Journal of Near-Death Studies, 8, 151 - 161.

Greyson, B. (1999). Defining the near-death experience. Mortality, 4, 7-19.

Greyson, B. (2001). Near-death experiences. In E. Cardena, \& S. J. Lynn (Eds.), Varieties of anomalous experience: Examining the scientific evidence (pp. 315 -352). Washington, DC: American Psychological Association.

Greyson, B., \& Stevenson, I. (1980). The phenomenology of near-death experiences. American Journal of Psychiatry, 137, 1193-1196.

Houran, J., \& Lange, R. (1997). Hallucinations that comfort: Contextual mediation of deathbed visions. Perceptual and Motor Skills, 84, 1491 - 1504. 
Houran, J., \& Lange, R. (2001). A Rasch hierarchy of haunt and poltergeist experiences. Journal of Parapsychology, 65, 41 - 58.

Houran, J., Wiseman, R., \& Thalbourne, M. A. (2002). Perceptual-personality characteristics associated with naturalistic haunt experiences. European Journal of Parapsychology, 17, $17-34$.

Irwin, H. J. (1999). An introduction to parapsychology (3rd ed.). Jefferson, NC: McFarland.

Irwin, H. J. (2000). The disembodied self: An empirical study of dissociation and the out-of-body experience. Journal of Parapsychology, 64, 261 - 277.

Kellehear, A. (1996). Experiences near death: Beyond medicine and religion. New York: Oxford University Press.

Kelly, E. W., Greyson, B., \& Stevenson, I. (1999). Can experiences near death furnish evidence of life after death? Omega, 40, 513-519.

Knoblauch, H., Schmied, I., \& Schnettler, B. (2001). Different kinds of near-death experience: A report on a survey of near-death experiences in Germany. Journal of Near-Death Studies, $20,15-29$.

Lange, R., \& Houran, J. (1997). Death anxiety and the paranormal: The primacy of belief over experience. Journal of Nervous and Mental Disease, 185, 584-586.

Lange, R., \& Houran, J. (1998). Delusions of the paranormal: A haunting question of perception. Journal of Nervous and Mental Disease, 186, 637-645.

Lange, R., \& Houran, J. (1999). The role of fear in delusions of the paranormal. Journal of Nervous and Mental Disease, 187, 159-166.

Lange, R., Irwin, H. J., \& Houran, J. (2000). Top-down purification of Tobacyk's Revised Paranormal Belief Scale. Personality and Individual Differences, 29, 131 - 156.

Lange, R., Irwin, H. J., \& Houran, J. (2001). Objective measurement of paranormal belief: A rebuttal to Vitulli. Psychological Reports, 88, 641-644.

Lange, R., \& Thalbourne, M. A. (2002). Rasch scaling paranormal belief and experience: Structure and semantics of Thalbourne's Australian Sheep-Goat Scale. Psychological Reports, 91, $1065-1073$.

Lange, R., Thalbourne, M. A., Houran, J., \& Storm, L. (2000). The Revised Transliminality Scale: Reliability and validity data from a Rasch top-down purification procedure. Consciousness and Cognition, 9, $591-617$.

Lester, D. (2000). Major dimensions of near-death experiences. Psychological Reports, 87, $835-836$.

Lester, D. (2003). Depth of near-death experiences and confounding factors. Perceptual and Motor Skills, 96, 18.

Linacre, J. M. (2001). A user's guide to Facets Rasch measurement computer program. Chicago: MESA Press.

Linacre, J. M., \& Wright, B. D. (1998). A user's guide to Winsteps, Bigsteps, and Ministeps: Raschmodel computer programs. Chicago: Mesa Press.

Lundahl, C. R. (1993). The near-death experience: A theoretical summarization. Journal of NearDeath Studies, 12, 105-118.

Masters, G. N. (1982). A Rasch model for partial credit scoring. Psychometrika, 47, 149-174.

McCutcheon, L. E., Lange, R., \& Houran, J. (2002). Conceptualization and measurement of celebrity worship. British Journal of Psychology, 93, 67-87.

Moody, R. A. (1975). Life after life. Covington, GA: Mockingbird.

Parker, A. (2001). What can cognitive psychology and parapsychology tell us about near-death experiences? Journal of the Society for Psychical Research, 65, 225-240.

Pope, J. E. (1991). Near-death experiences and attitudes towards life, death and suicide. Unpublished master's thesis, University of New England, Armidale, Australia.

Pope, J. E. (1994). Near-death experiences and attitudes towards life, death and suicide. Australian Parapsychological Review, 19, 23-26.

Rasch, G. (1980). Probabilistic models for some intelligence and attainment tests. Chicago: MESA Press (original published 1960). 
Ring, K. (1980). Life at death: A scientific investigation of the near-death experience. New York: Coward, McCann \& Geoghegan.

Sabom, M. B. (1982). Recollections of death: A medical investigation. New York: Harper \& Row. Stevenson, I., Cook, E. W., \& McClean-Rice, N. (1989). Are persons reporting 'near-death experiences' really near death? A study of medical records. Omega, 20, 45-54.

Wright, B. D., \& Masters, G. N. (1982). Rating scale analysis. Chicago: MESA Press.

Received 21 October 2002; revised version received I 8 April 2003

\section{Appendix}

\section{Near-Death Experience scale (Greyson, 1983)}

(1) Did time seem to speed up or slow down?

$0=$ No

$1=$ Time seemed to go faster or slower than usual

2 = Everything seemed to be happening at once; or time stopped or lost all meaning

(2) Were your thoughts speeded up?

$0=$ No

$1=$ Faster than usual

$2=$ Incredibly fast

(3) Did scenes from your past come back to you?

$0=$ No

$1=\mathrm{I}$ remembered many past events

2 = My past flashed before me, out of my control

(4) Did you suddenly seem to understand everything?

$0=$ No

$1=$ Everything about myself or others

$2=$ Everything about the universe

(5) Did you have a feeling of peace or pleasantness?

$0=$ No

$1=$ Relief or calmness

$2=$ Incredible peace or pleasantness

(6) Did you have a feeling of joy?

$0=$ No

$1=$ Happiness

$2=$ Incredible joy

(7) Did you feel a sense of harmony or unity with the universe?

$0=$ No

$1=$ I felt no longer in conflict with nature

$2=$ I felt united or one with the world

(8) Did you see, or feel surrounded by, a brilliant light?

$0=\mathrm{No}$

$1=$ An unusually bright light

$2=$ A light clearly of mystical or other-worldly origin

(9) Were your senses more vivid than usual?

$0=$ No

$1=$ More vivid than usual

$2=$ Incredibly more vivid 
(10) Did you seem to be aware of things going on elsewhere, as if by ESP?

$0=\mathrm{No}$

$1=$ Yes, but the facts have not been checked out

$2=$ Yes, and the facts have been checked out

(11) Did scenes from the future come to you?

$0=$ No

$1=$ Scenes from my personal future

$2=$ Scenes from the world's future

(12) Did you feel separated from your body?

$0=$ No

$1=\mathrm{I}$ lost awareness of my body

$2=$ I clearly left my body and existed outside it

(13) Did you seem to enter some other, unearthly world?

$0=\mathrm{No}$

$1=$ Some unfamiliar and strange place

$2=$ A clearly mystical or unearthly realm

(14) Did you seem to encounter a mystical being or presence, or hear an unidentifiable voice?

$0=\mathrm{No}$

$1=\mathrm{I}$ heard a voice I could not identify

$2=$ I encountered a definite being, or a voice clearly of mystical or unearthly origin

(15) Did you see deceased or religious spirits?

$0=\mathrm{No}$

$1=\mathrm{I}$ sensed their presence

$2=\mathrm{I}$ actually saw them

(16) Did you come to a border or point of no return?

$0=\mathrm{No}$

$1=$ I came to a definite conscious decision to return to life

2 = I came to a barrier that I was not permitted to cross; or was sent back against my will 